\title{
Logic-Based Outer Approximation for the Design of Discrete-Continuous Dynamic Systems with Implicit Discontinuities
}

\author{
Rubén Ruiz-Femenia ${ }^{a *}$, Jose A. Caballero ${ }^{a}$, Ignacio E. Grossmann ${ }^{\mathrm{b}}$ \\ ${ }^{a}$ Department of Chemical Engineering, University of Alicante, Ap. 99, Alicante. Spain. \\ ${ }^{b}$ Department of Chemical Engineering, Carnegie Mellon University. 5000 Forbes \\ Avenue. 15213, Pittsburgh, PA. USA. \\ Ruben.Ruiz@ua.es
}

\begin{abstract}
We address the optimization of discrete-continuous dynamic optimization problems using a disjunctive multistage modeling framework, with implicit discontinuities, which increases the problem complexity since the number of continuous phases and discrete events is not known a-priori. After setting a fixed alternative sequence of modes, we convert the infinite-dimensional continuous mixed-logic dynamic (MLDO) problem into a finite dimensional discretized GDP problem by orthogonal collocation on finite elements. We use the Logic-based Outer Approximation algorithm to fully exploit the structure of the GDP representation of the problem. This modelling framework is illustrated with an optimization problem with implicit discontinuities (diver problem).
\end{abstract}

Keywords: Logic-Based Outer Approximation, Discrete-Continuous Dynamic Systems, Mixed-Logic Dynamic Optimization, GDP, Orthogonal collocation method

\section{Introduction}

Many chemical process systems of practical interest are subject to discrete events that cause discontinuities in their dynamics. Dynamic models are required for batch and semi-batch processes which are inherently transient; for the operation of continuous processes in transient phases, including start-ups (Mynttinen and Li, 2012), shut-downs and changeovers from one to another steady state; and for safety analysis (LoteroHerranz and Galán, 2013). Optimization of discrete-continuous dynamic problems (also referred as hybrid systems) requires the treatment of non-smooth conditions within the problem formulation. These problems can be formulated as mixed integer nonlinear programing (MINLP) models, that allow to handle logic conditions that lead to nonsmoothness. However, the associated computational expense may be high for large systems with many discrete decisions. This is often the case in hybrid systems that can switch at any time. Raman and Grossmann (1994) developed the Generalized Disjunctive Programming (GDP), as an alternative modeling framework to the traditional mixed-integer formulations. The development of GDP has led to customized algorithms that exploit the disjunctive structure of the model. In particular, Turkay and Grossmann (1996) extended the outer approximation (OA) algorithm. We address the optimization of discrete-continuous dynamic optimization problems using a disjunctive multistage modeling framework that contains Boolean variables associated to alternative sets of differential equations for each stage (or continuous phase), and where switching from one continuous phase to the next occurs at some unknown time (implicit discontinuities). Before applying the logic-based OA algorithm, we transform the 
differential into algebraic equations by orthogonal collocation on finite elements.

\section{Mathematical problem formulation}

\subsection{Disjunctive multistage model}

The continuous phase of a process occurs in the time interval between two discrete events. When the process can reside in more than one mode for each stage, the dynamic system is described by an alternative sequence (Figure 1).

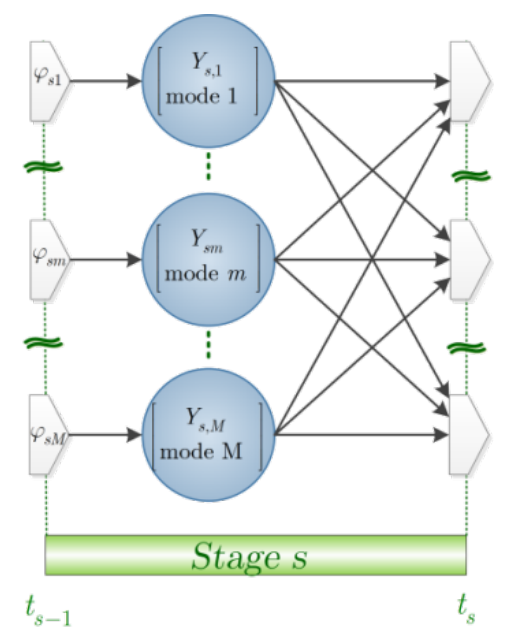

Figure 1. Alternative modes for each stage.

The first step is to extract a set of potential fixed direct sequences, which can be merged into one single fixed alternative sequence (i.e., the superstructure). A bypass stage maps the state variable values of one existing stage to the next. For further details on the general multistage modeling framework consult (Oldenburg and Marquardt, 2008).

\subsection{Discretization using orthogonal collocation}

We transform the disjunctive multistage problem into a discretized GDP problem by orthogonal discretization, a simultaneous method that fully discretizes the DAE system by approximating the control and state variables as piecewise polynomials functions over finite elements (Kameswaran and Biegler, 2006). Figure 2 shows how the time horizon is discretized. Accordingly, at each collocation point the state variable is represented by:

$x_{s i k}=x_{s i}^{0}+h_{s i} \sum_{j=1}^{K} \Omega_{j}\left(\tau_{k}\right) \dot{x}_{s i j}, s=1, \ldots, S, \quad i=1, \ldots, I, \quad k=1, \ldots, K$

where $x_{s i}^{0}$ is the value of the state variable at the beginning of element $i$ in stage $s, \dot{x}_{s i j}$ is the value of its first derivative in element $i$ at the collocation point $k$ in stage $s, h_{i s}$ is the length of element $i$ in stage $s, \Omega_{j}\left(\tau_{k}\right)$ is the interpolation polynomial of order $K$ for collocation point $j$, and $\tau_{k}$ is the non-dimensional time coordinate. We enforce continuity of the state variable across finite element boundaries in each stage by $x_{s i}^{0}=x_{s, i-1, K}$ for all $s=1, \ldots, S, i=2, \ldots, I$. Additional stage transition conditions map the differential state variable values across the stage boundaries:

$x_{s 1}^{0}-x_{s-1, I, K}=0, \quad s=2, \ldots, S$ 


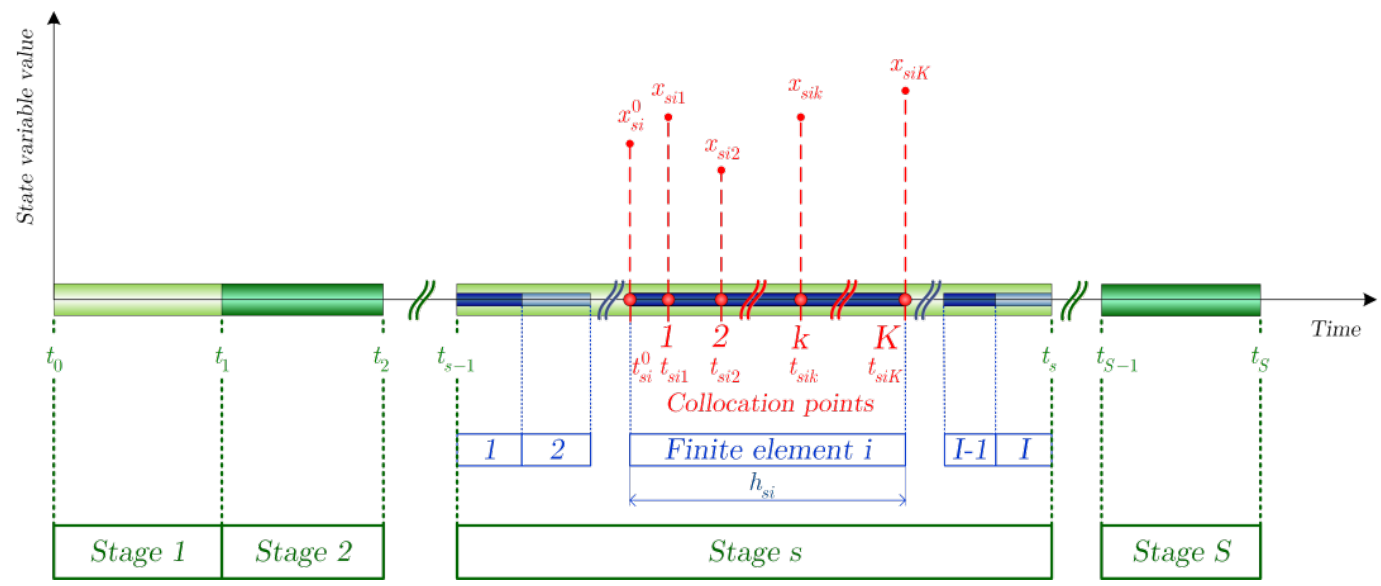

Figure 2. Discretization scheme used to apply the orthogonal collocation method.

For each stage, the collocation method requires the time to be discretized over each finite element at the selected collocation points $t_{s i k}$ :

$t_{s i k}=t_{s i}^{0}+h_{s i} \tau_{k}, s=1, \ldots, S, \quad i=1, \ldots, I, \quad k=1, \ldots, K$

where $t_{s i}^{0}$ is the value of the time at the beginning of element $i$ in stage s. Time continuity between stages and between elements within a stage is also enforced by the following constraints:

$\begin{aligned} t_{s, 1}^{0} & =t_{s-1, I, K}, \quad s=2, \ldots, S \\ t_{s i}^{0} & =t_{s, i-1, K}, \quad s=1, \ldots, S, \quad i=2, \ldots, I\end{aligned}$

\section{Case study}

The proposed modeling framework has been assessed with a benchmark case study, the diver problem (Barton, Allgor, 1998). The design task is to calculate the depth-time profile of a scuba diver who wishes to collect an item from the ocean floor with the minimum consumption of air. To prevent decompression sickness, the diver can make safety stops of 4 minutes during the ascent. The unknown switching structure arises from the number of decompression stops that the diver has to make during the ascent. The model comprises three state variables, which are the pressure in the tank $P^{\operatorname{tank}}(t)$, the surrounding pressure $P(t)$ and the partial pressure of $N_{2}$ in the tissue $P^{\text {tissue }}(t)$. The control variable is the velocity of the descent/ascend of the scuba diver, $u(t)$ :

$$
\begin{array}{lc}
\max _{u(t)} & P^{\text {tank }}\left(t^{\text {final }}\right) \\
\text { s.t. } & \frac{d P^{\text {tank }}}{d t}(t)=\frac{-q}{V_{\text {tank }}} P(t) \\
& \frac{d P^{\text {tissue }}}{d t}(t)=\frac{\ln 2}{5}\left(0.79 P(t)-P^{\text {tissue }}(t)\right) \\
& \frac{d P}{d t}(t)=-1 \times 10^{5} \rho g u(t)
\end{array}
$$

Ascent mode: $\underline{u} \leq u(t) \leq \bar{u}$

Switch to decompression mode if: $\quad P^{\text {tissue }}(t) \geq 2 \times 0.79 P(t)$

Decompression mode: $u(t)=0$ and wait for 4 min and then switch to ascent mode.

\subsection{Mixed-Logic Dynamic Optimization formulation}


We formulate a disjunctive multistage representation of problem (5) by a fixing alternative sequence for a certain number of stages that may include a bypass term in the disjunction of any stage. Particularly, we use a fixed alternative sequence with three decompression stops. Hence, the MLDO problem is stated as:

$$
\max _{u_{s}(t), f^{\text {final }}, t^{\text {bottom }}}=P_{S}^{\text {tank }}\left(t_{S}\right)
$$

s.t. $P\left(t_{0}\right)=1, P\left(t^{\text {bottom }}\right)=P\left(t_{1}\right)=6, P\left(t^{\text {final }}\right)=P\left(t_{S}\right)=1$

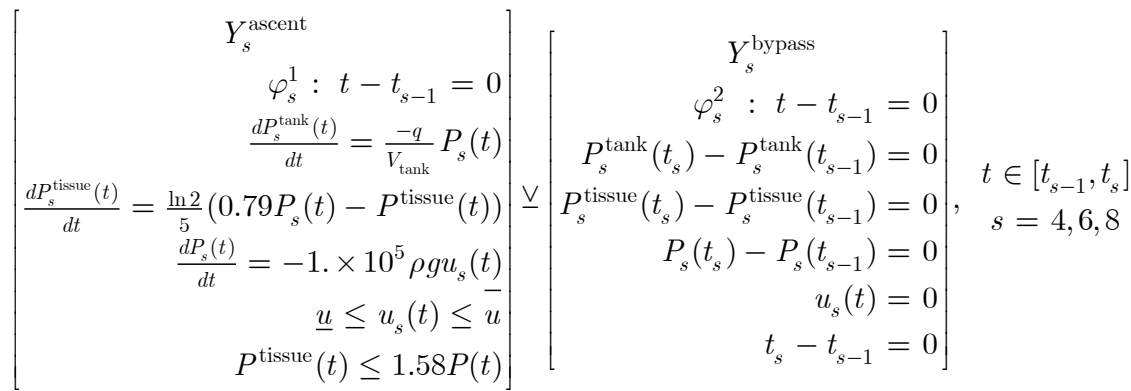

$$
\begin{aligned}
& \left.\left[\begin{array}{r}
Y_{s}^{\text {deco }} \\
\varphi_{s}^{1}: P^{\text {tissue }}(t)-\theta 0.79 P(t)=0 \\
\frac{d P_{s}^{\text {tank }}(t)}{d t}=\frac{-q}{V_{\text {tank }}} P_{s}(t) \\
\frac{d P_{s}^{\text {tisue }}(t)}{d t}=\frac{\ln 2}{5}\left(0.79 P_{s}(t)-P^{\text {tissue }}(t)\right) \\
\frac{d P_{s}(t)}{d t}=-1 \times 10^{5} \rho g u_{s}(t) \\
u_{s}(t)=0 \\
t_{s}-t_{s-1}=4
\end{array}\right] \underline{Y_{s}^{\text {bypass }}} \begin{array}{r}
\varphi_{s}^{2}: t-t_{s-1}=0 \\
P_{s}^{\text {tank }}\left(t_{s}\right)-P_{s}^{\text {tank }}\left(t_{s-1}\right)=0 \\
P_{s}^{\text {tissue }}\left(t_{s}\right)-P_{s}^{\text {tissue }}\left(t_{s-1}\right)=0 \\
P_{s}\left(t_{s}\right)-P_{s}\left(t_{s-1}\right)=0 \\
u_{s}(t)=0 \\
t_{s}-t_{s-1}=0
\end{array}\right], \quad t \in\left[t_{s-1}, t_{s}\right. \\
& Y_{s}^{\text {ascent }}, s=1,2 \therefore Y_{s}^{\text {deco }} \Leftrightarrow Y_{s+1}^{\text {ascent }}, s=3,5,7
\end{aligned}
$$

\subsection{MLDO Discretization}

The time continuous MLDO problem is converted into a finite dimensional discretized GDP by a full orthogonal collocation of the three state variables using Eqs. (1)-(4):

$$
\min _{\left(P_{s i k}^{\text {tank }}, P_{s i}^{\text {tank }, 0}, \dot{P}_{s i k}^{\text {tank }}, P_{s i k}^{\text {tisue }}, P_{s i}^{\text {tisue }, 0}, \dot{P}_{s i k}^{\text {tisue }}, P_{s i k}, P_{s i}^{0}, \dot{S}_{s i k}, h_{s i}, u_{s}\right)} Z=-P_{S I K}^{\text {tank }}
$$

s.t., Time discretization: $t_{s i k}=t_{s i}^{0}+h_{s i} \tau_{k}, s=1, \ldots, S, i=1, \ldots, I, k=1, \ldots, K$

Time continuity finite element: $t_{s i}^{0}=t_{s, i-1, K}, s=1, \ldots, S, \quad i=2, \ldots, I$

Time mapping: $t_{s i}^{0}=t_{s-1, I, K}, s=2, \ldots, S$

$$
\begin{gathered}
P_{s i k}^{\text {tank }}=P_{s i}^{\text {tank }, 0}+h_{s i} \sum_{j=1}^{K} \Omega_{j k} \dot{P}_{s i k}^{\text {tank }} \\
\text { State discretization: } P_{s i k}^{\text {tissue }}=P_{s i}^{\text {tissue }, 0}+h_{s i} \sum_{j=1}^{K} \Omega_{j k} \dot{P}_{s i k}^{\text {tissue }} \\
P_{s i k}=P_{s i}^{0}+h_{s i} \sum_{j=1}^{K} \Omega_{j k} \dot{P}_{s i k} \\
P_{s, 1}^{0}-P_{s-1, I, K}=0 \\
\text { Time mapping: } \left.\left.\begin{array}{c}
P_{s, 1}^{\text {tank }, 0}-P_{s-1, I, K}^{\text {tank }}=0 \\
P_{s, 1}^{\text {tissue } 0}-P_{s-1, I, K}^{\text {tisue }}=0
\end{array}\right\}, \begin{array}{c}
i=1, \ldots, I \\
k=1, \ldots, K
\end{array}\right\}, s=2, \ldots, S
\end{gathered}
$$


$\left.\begin{array}{rl}P_{s i}^{\mathrm{tank}, 0} & =P_{s, i-1, K}^{\mathrm{tank}} \\ \text { State continuity finite element: } P_{i}^{\text {tissue }, 0} & =P_{i-1, K}^{\text {tisse }} \\ P_{i}^{0} & =P_{i-1, K}\end{array}\right\}, s=1, \ldots, S, i=2, \ldots, I$

Common end point constraints: $P_{1, I, K}=6, P_{S, I, K}=1$
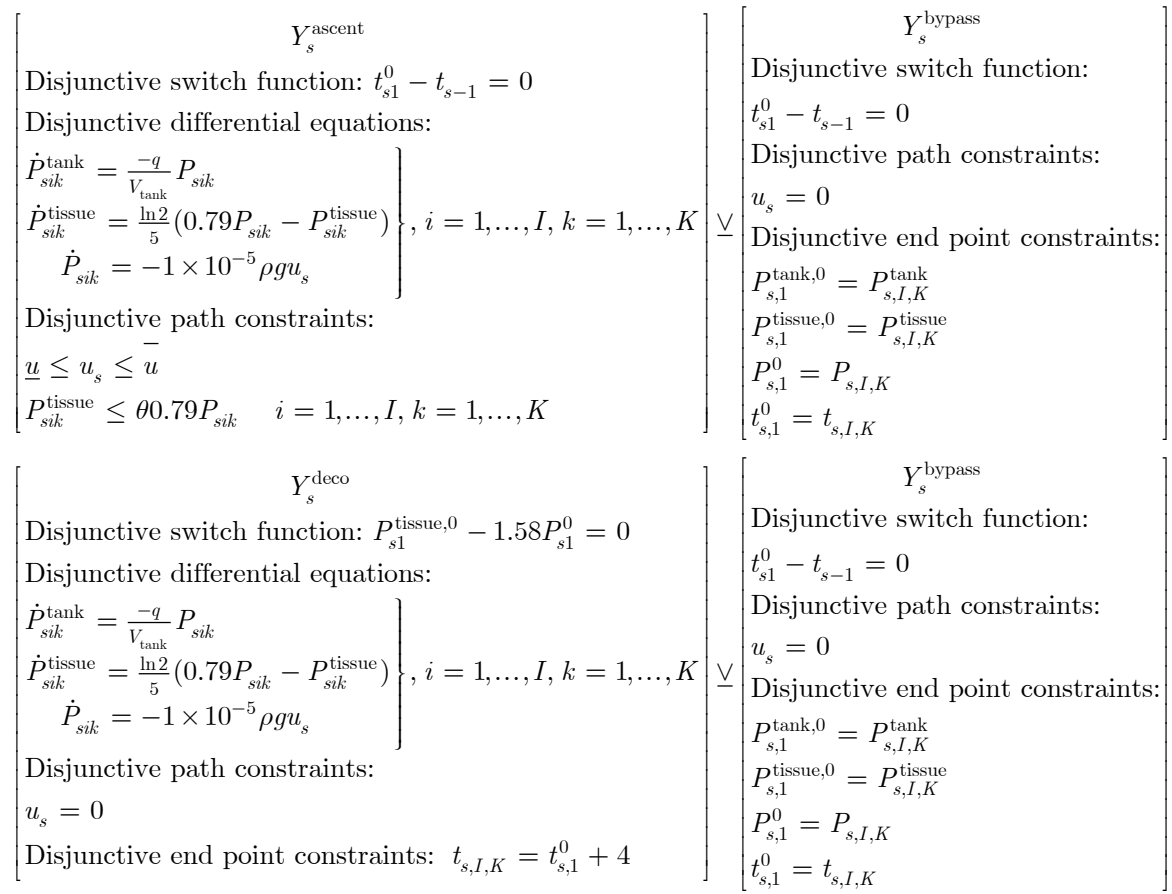

$Y_{s}^{\text {ascent }}, s=1,2, \therefore Y_{s}^{\text {deco }} \Leftrightarrow Y_{s+1}^{\text {ascent }}, s=3,5,7$

\subsection{Logic-based discretized NLP subproblem}

The NLP subproblem is obtained by fixing the values of the Boolean variables $Y_{s m}$. Due to space limitations we omit stating the full discretized NLP subproblem.

\subsection{Logic-based discretized Master problem}

We formulate the discretized Master problem with the accumulated linearizations of the nonlinear constraints from previous solutions of each discretized NLP subproblem. For the shake of shortness, we exclude the representation of the discretized Master problem.

\section{Results and discussion}

The problem is solved by the logic based OA algorithm implemented in GAMS using the CONOPT solver for the NLP subproblems and the CPLEX 12.5.1.0 solver for MILP master problems. Our GAMS implementation allows writing disjunctions with more than two terms. The discretized linear master problem is reformulated as an MILP using the Hull Reformulation (HR). Figure 3 shows the optimal results of this case study, where we discretize the time domain using 3 collocation points per finite element. 


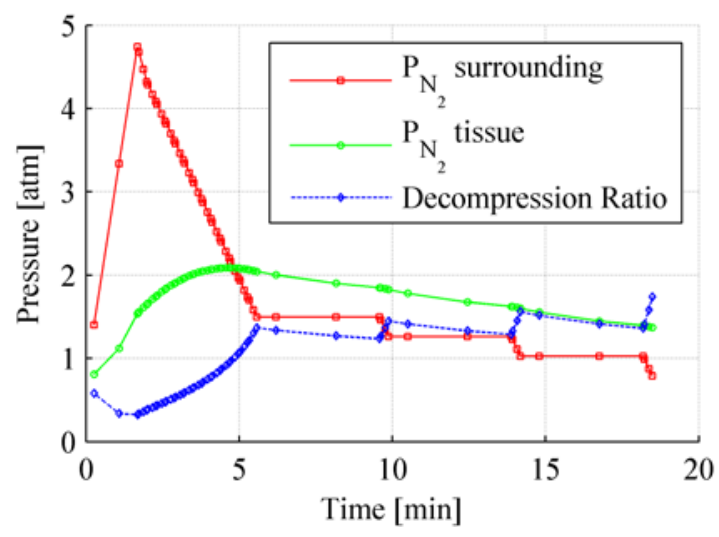

Figure 3. Optimal Pressure profiles.

\section{Conclusions}

The GDP modeling framework proposed coupled with logic-based OA algorithm can tackle with the optimization of continuous dynamic systems with implicit discontinuities. The methodology requires creating a superstructure with a certain number of stages and a fixed alternative sequence of modes. The application of the logic-based OA algorithm reduces the problem size in comparison when the model is directly reformulated into an MINLP, due to the differential equations corresponding to the non-active modes for a particular stage are discarded. Further work extends the proposed modeling framework to a multistage batch distillation process and the combination of scheduling with dynamic optimization.

\section{Acknowledgements}

The authors acknowledge financial support from «Estancias de movilidad en el extranjero "Jose Castillejo" (JC2011-0054)» of the Spanish "Ministerio de Educación”, and from the Spanish "Ministerio de Ciencia e Innovacion” (CTQ2012-37039-C02-02).

\section{References}

I. Mynttinen, P. Li, 2012, A stop-and-restart approach to hybrid dynamic optimization problems, Computer Aided Chemical Engineering, Volume 30, 822-826.

I. Lotero-Herranz, S. Galán, 2013, Automated HAZOP using hybrid discrete/continuous process models, Computer Aided Chemical Engineering, Volume 32, 991-996.

R. Raman, I.E. Grossmann, 1994, Modelling and computational techniques for logic based integer programming, Computers and Chemical Engineering, 18, 7, 563-578.

M. Türkay, I.E. Grossmann, 1996, Logic-based MINLP algorithms for the optimal synthesis of process networks, Computers and Chemical Engineering, 20, 8, 959-978.

J. Oldenburg, W. Marquardt, 2008, Disjunctive modeling for optimal control of hybrid systems, Computers and Chemical Engineering, 32, 10, 2346-2364.

S. Kameswaran, L.T. Biegler, 2006, Simultaneous dynamic optimization strategies: Recent advances and challenges, Computers and Chemical Engineering, 30, 10-12, 1560-1575.

P.I. Barton, R.J. Allgor, W.F. Feehery, S. Galán, 1998, Dynamic optimization in a discontinuous world, Industrial and Engineering Chemistry Research, 37, 3, 966-981. 